\title{
Acquired Ocular Motor Nerve Palsy in Neurology Clinics: A Prospective Multicenter Study
}

\author{
Kwang-Dong Choi ${ }^{\mathrm{a}}$ \\ Seo Young Choi ${ }^{\mathrm{a}}$, Ji-Soo Kim ${ }^{\mathrm{b}}$ \\ Jae-Hwan Choic, Tae-Ho Yang ${ }^{d}$ \\ Sun-Young $\mathrm{Oh}^{d}$, Sung-Hee Kim ${ }^{\mathrm{e}}$ \\ Hak-Seung Lee, Seung-Han Lee ${ }^{g}$ \\ Seong-Hae Jeong ${ }^{\text {h }}$ \\ Hyo-Jung Kim ${ }^{\text {b, }}$ \\ Jeong-Yoon Choi ${ }^{\text {b }}$
}

aDepartment of Neurology, Pusan National University Hospital, Pusan National University School of Medicine and

Biomedical Research Institute, Busan,

Korea

'Department of Neurology, Seoul National University College of Medicine, Seoul

National University Bundang Hospital, Seongnam, Korea

'Department of Neurology, Pusan National University Yangsan Hospital, Pusan

National University School of Medicine and Biomedical Research Institute,

Yangsan, Korea

${ }^{\mathrm{d} D e p a r t m e n t}$ of Neurology, Chonbuk

National University School of Medicine,

Jeonju, Korea

eDepartment of Neurology, Kyungpook

National University School of Medicine,

Daegu, Korea

fDepartment of Neurology, Wonkwang

University School of Medicine, Institute of

Wonkwang Medical Science and

Regional Cardiocerebrovascular Center,

Iksan, Korea

${ }^{9}$ Department of Neurology, Chonnam

National University Medical School,

Chonnam National University Hospital,

Gwangju, Korea

hDepartment of Neurology, Chungnam

National University School of Medicine,

Daejeon, Korea

'Research Administration Team, Seoul

National University Bundang Hospital,

Seongnam, Korea

Received October 10, 2018

Revised December 3, 2018

Accepted December 3, 2018

\section{Correspondence}

Ji-Soo Kim, MD, PhD

Department of Neurology,

Seoul National University

College of Medicine,

Seoul National University

Bundang Hospital,

82 Gumi-ro 173beon-gil, Bundang-gu,

Seongnam 13620, Korea

Tel +82-31-787-7463

Fax +82-31-719-6828

E-mail jisookim@snu.ac.kr
Background and Purpose This study aimed to determine the patterns and etiologies of acquired ocular motor nerve palsy (OMNP) diagnosed in neurology clinics. We also investigated the clinical features that may predict the causes other than microvascular ischemia in isolated OMNP.

Methods We performed a prospective multicenter study that had recruited 298 patients with acquired OMNP from the neurology clinics of referral-based 9 university hospitals in Korea. We finally selected 235 patients with isolated OMNP and divided them into older (age $\geq 50$ years, $n=188$ ) and younger (age $<50$ years, $n=47$ ) groups. We investigated the underlying etiologies of acquired OMNP. We also estimated the frequency of microvascular ischemia and other causes in isolated OMNP, and sought to determine the clinical features that can predict the causes other than microvascular ischemia.

Results Abducens nerve palsy was the most common (40\%) of the acquired OMNPs, followed by oculomotor nerve palsy (27\%), trochlear nerve palsy (23\%), and multiple OMNPs (10\%). The etiologies included microvascular ischemia (47\%), inflammatory (21\%), stroke (5\%), trauma (5\%), neoplasm (3\%), and others ( $2 \%$ ), with the cause not being determined in $13 \%$ of the patients. Microvascular ischemia was the most common cause $(83 \%)$ in patients aged $\geq 50$ years with isolated OMNP, followed by inflammation (6\%), stroke (6\%), neoplasm (3\%), and aneurysm (1\%). Microvascular ischemia was more common in the older than the younger group ( $83 \%$ vs. $49 \%, p<0.001$ ). The intensity of headache was the only risk factor for causes other than microvascular ischemia in isolated OMNP.

Conclusions Vascular and inflammatory disorders are the most common causes of acquired OMNP diagnosed in neurology clinics. Microvascular ischemia was the predominant cause of isolated OMNP. Severe headache indicates causes other than microvascular ischemia in isolated OMNP.

Key Words acquired ocular motor nerve palsy, isolated ocular motor nerve palsy, diplopia, microvascular ischemia.

\section{INTRODUCTION}

Acquired ocular motor nerve palsy (OMNP) is a common cause of acute diplopia in neurological practice. ${ }^{1-4}$ Acquired OMNP can result from various causes including trauma, intracranial tumors, stroke, aneurysm, and microvascular ischemia. ${ }^{1-4}$ Prompt diagnosis and treatment are important in acquired OMNP since it can lead to death if appropriate treatment is not applied. ${ }^{5-8}$ The largest series from the Mayo Clinic that included 4,278 patients identified abducens nerve palsy as the most common type of OMNP (44\%), but also the cause could not be determined in $25 \%$ of cases. ${ }^{9}$ Other studies have found various etiologies depending on the included population and the criteria used to determine the eti-

(c) This is an Open Access article distributed under the terms of the Creative Commons Attribution Non-Commercial License (https://creativecommons.org/licenses/by-nc/4.0) which permits unrestricted non-commercial use, distribution, and reproduction in any medium, provided the original work is properly cited. 
ology ${ }^{10-17}$ However, most studies of acquired OMNPs have been limited by their retrospective designs, being performed only in ophthalmology clinics, and the nonavailability of advanced neuroimaging techniques that can reliably detect small intra-axial lesions.

A few recent studies have prospectively assessed the underlying etiologies of isolated OMNP, and the efficacy of emergency neuroimaging. ${ }^{18-21}$ Isolated OMNP in patients aged $\geq 50$ years frequently occurs from presumed microvascular ischemia of the nerve in the presence of vascular risk factors, while the reported frequency of causes other than microvascular ischemia has ranged from $4 \%$ to $17 \% .{ }^{19-21}$ However, there has been no prospective study of an Asian population with isolated OMNP, and no study has investigated the clinical features for guiding neuroimaging with the inclusion of a control arm.

We conducted a prospective multicenter study to investigate the underlying etiologies of acquired OMNP in neurology clinics. We also attempted to estimate the frequency of microvascular ischemia and other causes in a Korean population with isolated OMNP, and sought to determine the clinical features that may predict causes other than microvascular ischemia.

\section{METHODS}

\section{Study design and participants}

We performed a prospective multicenter study that recruited 298 patients with acquired OMNP from the neurology clinics of 9 university hospitals in Korea between January and December 2015. The inclusion criteria were 1) acquired binocular diplopia within 30 days of onset and 2) diplopia due to OMNP. Exclusion criteria were a prior history of strabismus, orbital diseases, congenital OMNP, diplopia due to extraocular muscle disorders, neuromuscular junction (NMJ) disorders, nuclear or supranuclear disorders, inability to undergo MRI, history of neurological diseases that can influence cognitive function, or incomplete data.

During the study period, 412 patients with acquired binocular diplopia were initially recruited. After excluding 114 patients with nuclear or supranuclear palsy $(n=46), \mathrm{NMJ}$ disorders ( $n=27)$, other causes $(n=21)$, extraocular muscle disorders $(n=11)$, and incomplete data $(n=9), 298$ patients with acquired OMNP were finally selected for this study (Fig. 1).

We defined isolated OMNP as the absence of neurological signs and symptoms other than headache or periorbital pain. Among 298 patients with acquired OMNP, we further selected 235 with isolated OMNP after excluding 63 patients with a history of preceding head trauma, multiple OMNPs, or other neurological symptoms and signs. We divided the

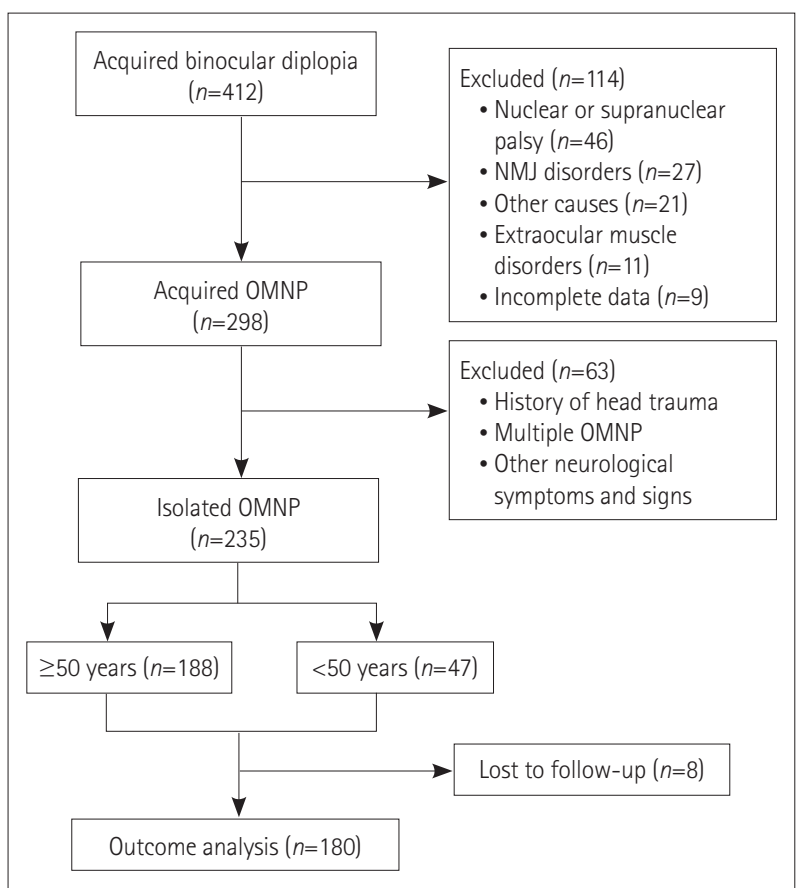

Fig. 1. Study flow diagram. Initially, 412 patients with acquired binocular diplopia were recruited, and 298 with acquired OMNP were selected for this study after excluding those with nuclear or supranuclear palsy, neuromuscular junction disorders, other causes, extraocular muscle disorders, and incomplete data. Among 298 patients with acquired OMNP, we further selected 235 with isolated OMNP after excluding patients with a history of preceding head trauma, multiple OMNPs, or other neurological symptoms and signs. We divided the patients with isolated OMNP into two groups according to their age: $<50$ years and $\geq 50$ years. The outcome analysis was applied to 180 patients with isolated OMNP. NMJ: neuromuscular junction, OMNP: ocular motor nerve palsy.

patients with isolated OMNP into two groups according to their age: $<50$ years $(n=47)$ and $\geq 50$ years $(n=188)$.

We collected data using a structured registration form that included demographic features, clinical characteristics (the frequency and intensity of headache, ocular pain, neurological signs, vascular risk factors, and a history of previous infection, head trauma, or cancer), the results of radiological and laboratory evaluations, treatments, and prognosis.

This study followed the tenets of the Declaration of Helsinki and was approved by Institutional Review Board of Pusan National University Hospital (approval no. 1605-001041). Informed contents were obtained after the nature and possible consequences of this study had been explained to the participants.

\section{Diagnostic procedure}

All participants underwent imaging of the brain and orbit with MRI including diffusion-weighted imaging (DWI) and MR angiography. The laboratory evaluation included com- 
plete blood counts, routine chemistry, thyroid function tests, antithyroid antibodies, serum anti-acetylcholine-receptor antibody, repetitive nerve stimulation test, and neostigmine test to exclude extraocular muscle or NMJ disorders. Vascular risk factors were defined: aged $\geq 60$ years, hypertension, diabetes mellitus, smoking, previous stroke or myocardial infarction, coronary artery disease, and dyslipidemia. We assessed the serum anti-GQ1b antibody level in patients with antecedent infection or decreased deep tendon reflexes, and performed CSF analysis in patients with accompanying headache.

Using the previously reported criteria for the etiologies of ophthalmoplegia, the cause of each type of acquired OMNP was assigned according to the medical history, results of radiological and laboratory evaluations, and clinical course. ${ }^{5,9,17,22}$ Appropriate lesions should have been documented in neuroimaging to diagnose cerebral infarctions, brain tumors, inflammation, and vascular disorders. A diagnosis of TolosaHunt syndrome was based on the 2018 criteria of the International Headache Society. ${ }^{23}$ A diagnosis of microvascular ischemia required no alternative cause in MRI and clinical tests, the presence of at least one vascular risk factor, and spontaneous resolution. When there were no identifiable causes, the etiology was classified as undetermined.

In patients with isolated OMNP, we used the recently reported criteria for a diagnosis of presumed microvascular ischemia that was assigned to those patients for whom MRI and clinical testing did not reveal an alternative cause, other neurological signs remained absent, and the ophthalmoparesis resolved spontaneously. ${ }^{19,21}$

\section{Treatment strategy and outcome measurement}

Patients with isolated OMNP due to other causes received conservative, medical, or surgical treatments according to the underlying etiologies, while the remaining patients with microvascular ischemia were followed up without receiving any specific treatment.

All of the patients who were diagnosed with isolated OMNP were followed up for at least 6 months, and the outcome was considered as resolved when both the tropia and diplopia disappeared.

\section{Statistical analysis}

A logistic regression analysis was used to assess the independent predictors of other causes in patients with isolated OMNP. Variables with $p<0.05$ in the univariate analysis were considered to represent explanatory variables. Chi-square or Fisher's exact tests were used to compare the proportion of men, each OMNP, and etiologies between the younger (age $<50$ years) and older ( $\geq 50$ years) patients. Statistical analyses were performed using SPSS (version 18.0, SPSS Inc., Chicago, IL, USA), and $p<0.05$ was considered significant.

\section{RESULTS}

The patients with acquired OMNP comprised 185 men (62\%) and 113 women $(38 \%)$ aged $58 \pm 15$ years (mean \pm SD), with a median age of 59 years and an age range of 9 to 88 years. The acquired OMNPs included abducens nerve palsy (40\%), oculomotor nerve palsy (27\%), trochlear nerve palsy (23\%), and multiple OMNPs (10\%) (Table 1). The etiologies of acquired OMNP were microvascular ischemia (47\%), inflammation (21\%), undetermined (13\%), stroke (5\%), trauma $(5 \%)$, neoplasm (3\%), others (2\%), carotid cavernous fistula (CCF, $2 \%$ ), and aneurysm (1\%). The causes of each OMNP were mainly microvascular ischemia, inflammation, and undetermined, but the frequencies of trauma and neoplasm were somewhat higher in trochlear nerve palsy and abducens

Table 1. Demography and etiologies of acquired OMNP

\begin{tabular}{lccccc}
\hline & III & IV & VI & Multiple & Total \\
\hline Patients, $n(\%)$ & $81(27)$ & $69(23)$ & $118(40)$ & $30(10)$ & $298(100)$ \\
Age, $y$, mean (SD) & $58(15)$ & $61(11)$ & $58(15)$ & $50(22)$ & $58(15)$ \\
Male, $n(\%)$ & $51(63)$ & $47(68)$ & $76(64)$ & $11(37)$ & $185(62)$ \\
Etiology, $n$ (\%) & & & & \\
$\quad$ Microvascular ischemia & $44(54)$ & $36(52)$ & $61(52)$ & $0(0)$ & $141(47)$ \\
Inflammation & $14(17)$ & $8(12)$ & $20(17)$ & $22(73)$ & $64(21)$ \\
Undetermined & $9(11)$ & $11(16)$ & $18(15)$ & $2(7)$ & $40(13)$ \\
Stroke & $8(10)$ & $2(3)$ & $6(5)$ & $0(0)$ & $16(5)$ \\
Trauma & $3(4)$ & $10(14)$ & $1(1)$ & $2(7)$ & $16(5)$ \\
Neoplasm & $1(1)$ & $0(0)$ & $8(7)$ & $1(3)$ & $10(3)$ \\
Others & $0(0)$ & $1(1)$ & $2(2)$ & $3(10)$ & $6(2)$ \\
CCF & $0(0)$ & $1(1)$ & $2(2)$ & $2(7)$ & $0(0)$ \\
Aneurysm & $2(2)$ & $0(0)$ & $0(0)$ & $2(1)$ & \\
\hline
\end{tabular}

CCF: carotid cavernous fistula, OMNP: ocular motor nerve palsy, III: oculomotor nerve palsy, IV: trochlear nerve palsy, VI: abducens nerve palsy. 
nerve palsy, respectively. The main cause of multiple OMNPs was inflammation (73\%).

The 188 patients aged 50 years with isolated OMNP included 122 men (64\%) and 66 women (36\%) aged $65 \pm 8$ years, with a median age of 64 years and an age range of 50 to 88 years. Isolated OMNP had an etiology of microvascular ischemia in $83 \%$ of cases, with the remaining $17 \%$ comprising inflammation (6\%), stroke (6\%), neoplasm (3\%), and aneurysm (1\%) (Table 2). Inflammatory disorders comprised

Table 2. Demography and etiologies of patients aged 50 years and older with isolated OMNP

\begin{tabular}{llllc}
\hline & \multicolumn{1}{c}{ III } & \multicolumn{1}{c}{ IV } & \multicolumn{1}{c}{ VI } & Total \\
\hline Patients, $n(\%)$ & $58(31)$ & $49(26)$ & $81(43)$ & $188(100)$ \\
\hline Age, $y$, mean (SD) & $64(9)$ & $65(8)$ & $59(8)$ & $65(8)$ \\
Male, $n(\%)$ & $36(62)$ & $34(69)$ & $52(64)$ & $122(64)$ \\
\hline Etiology, $n(\%)$ & & & & \\
\hline Microvascular ischemia & $43(74)$ & $43(88)$ & $70(86)$ & $156(83)$ \\
\hline Other causes & $15(26)$ & $6(12)$ & $11(14)$ & $32(17)$ \\
\hline$\quad$ Inflammation & 6 & 5 & 1 & 12 \\
\hline Stroke & 7 & 1 & 3 & 11 \\
\hline Neoplasm & 1 & 0 & 5 & 6 \\
\hline Aneurysm & 1 & 0 & 0 & 1 \\
\hline Vascular malformation & 0 & 0 & 1 & 1 \\
\hline Others & 0 & 0 & 1 & 1 \\
\hline
\end{tabular}

OMNP: ocular motor nerve palsy, III: oculomotor nerve palsy, IV: trochlear nerve palsy, VI: abducens nerve palsy.
Tolosa-Hunt syndrome $(n=7)$, pachymeningitis $(n=3)$, and demyelinating disease $(n=1)$. Inflammation and stroke were the most common other causes of isolated oculomotor and trochlear nerve palsies, while neoplasm and stroke were most frequent in abducens nerve palsy.

The presence [odds ratio $(\mathrm{OR})=2.9,95 \% \mathrm{CI}=1.3-6.3$ ] and a higher intensity of headache significantly increased the risk of causes other than microvascular ischemia in isolated OMNP, whereas the presence of vascular risk factors $(\mathrm{OR}=0.4,95 \%$ $\mathrm{CI}=0.2-0.9)$ was more likely to be associated with microvascular ischemia (Table 3). However, there were no differences in the frequency of a history of previous infection or cancer, pupillary involvement, pupil-sparing partial third-nerve palsy, or the degree (complete vs. partial) of external ophthalmoplegia between OMNP due to microvascular ischemia and OMNP due to other causes.

Almost all of the patients $(n=180)$ were followed up for at least 6 months. Most of them (149/180, 83\%) showed complete resolution within 6 months of the symptom onset with or without receiving treatment. Complete resolution was observed in $85 \%$ of OMNP cases due to microvascular ischemia and in 69\% of OMNP cases due to other causes (Table 4). The proportion of complete resolution did not differ between OMNP due to microvascular ischemia and OMNP due to other causes at 1,3 , or 6 months after the onset of symptoms.

Table 3. Comparison of clinical characteristics between patients with isolated OMNP due to microvascular ischemia and other causes

\begin{tabular}{|c|c|c|c|c|}
\hline & $\begin{array}{l}\text { Patients with other causes } \\
\qquad(n=32)\end{array}$ & $\begin{array}{l}\text { Patients with microvascular } \\
\text { ischemia }(n=156)\end{array}$ & OR $(95 \% \mathrm{Cl})$ & $p$ \\
\hline Age, $y$, mean (SD) & $63.7(10.6)$ & $65.3(8)$ & & 0.337 \\
\hline Male, $n(\%)$ & $16(50)$ & $106(68)$ & $0.5(0.2-1.0)$ & 0.053 \\
\hline \multicolumn{5}{|l|}{ Headache } \\
\hline Frequency, $n(\%)$ & $16(50)$ & $40(26)$ & $2.9(1.3-6.3)$ & 0.006 \\
\hline Intensity, VAS score, mean (SD) & $5.8(2.1)$ & $4.1(1.5)$ & & 0.001 \\
\hline Ocular pain, $n(\%)$ & $11(34)$ & $38(24)$ & $1.6(0.7-3.7)$ & 0.240 \\
\hline Complete paralysis (III and VI), $n(\%)$ & $8 / 26(31)$ & $32 / 113(28)$ & & $0.488^{*}$ \\
\hline Ptosis (III), $n(\%)$ & $13 / 15(87)$ & $29 / 43(67)$ & & $0.151^{*}$ \\
\hline Involvement of pupil (III), n (\%) & $3 / 15(20)$ & $6 / 43(14)$ & & $0.682^{*}$ \\
\hline Pupil-sparing partial paralysis (III), $n$ (\%) & $8 / 15(53)$ & $21 / 43(49)$ & & $0.764^{*}$ \\
\hline \multicolumn{5}{|l|}{ Vascular risk factors } \\
\hline Frequency, $n(\%)$ & $21(66)$ & $130(83)$ & $0.4(0.2-0.9)$ & 0.022 \\
\hline Mean number, mean (SD) & $2(1.3)$ & $1.9(1.1)$ & & 0.682 \\
\hline Preceding infection, $n(\%)$ & $1(3)$ & $21(14)$ & $0.2(0.0-1.6)$ & 0.132 \\
\hline History of cancer, $n(\%)$ & $4(13)$ & $11(7)$ & $2.8(0.6-6.3)$ & 0.291 \\
\hline \multicolumn{5}{|l|}{ Resolution, $n(\%)$} \\
\hline Within 1 month & $7 / 29(24)$ & 25/151 (17) & & $0.328^{*}$ \\
\hline Within 3 month & $19 / 29(31)$ & 96/151 (64) & & $0.842^{*}$ \\
\hline Within 6 month & 23/29 (79) & 129/151 (85) & & $0.405^{*}$ \\
\hline
\end{tabular}

${ }^{*}$ Chi-square tests were used due to different sample size.

OMNP: ocular motor nerve palsy, OR: odds ratio, VAS: visual analogue scale, III: oculomotor nerve palsy, VI: abducens nerve palsy. 
Table 4. Outcome of isolated ocular motor nerve palsy

\begin{tabular}{|c|c|c|c|c|c|c|}
\hline & \multicolumn{2}{|c|}{ Oculomotor nerve palsy } & \multicolumn{2}{|c|}{ Trochlear nerve palsy } & \multicolumn{2}{|c|}{ Abducens nerve palsy } \\
\hline & $\begin{array}{l}\text { Other causes } \\
\qquad(n=13)\end{array}$ & $\begin{array}{c}\text { Microvascular } \\
\text { ischemia }(n=41)\end{array}$ & $\begin{array}{l}\text { Other causes } \\
\qquad(n=6)\end{array}$ & $\begin{array}{c}\text { Microvascular } \\
\text { ischemia }(n=42)\end{array}$ & $\begin{array}{l}\text { Other causes } \\
\qquad(n=10)\end{array}$ & $\begin{array}{c}\text { Microvascular } \\
\text { ischemia }(n=68)\end{array}$ \\
\hline Complete resolution, $n(\%)$ & $10(77)$ & $34(83)$ & $1(17)$ & $36(86)$ & $9(90)$ & $59(87)$ \\
\hline
\end{tabular}

Table 5. Comparison of characteristics between patients aged 50 years or more and less than 50 years in isolated OMNP

\begin{tabular}{|c|c|c|c|}
\hline & Aged 50 years and older $(n=188)$ & Aged less than 50 years $(n=47)$ & $p$ \\
\hline Male, $n(\%)$ & $122(64)$ & $29(62)$ & 0.683 \\
\hline \multicolumn{4}{|l|}{ Frequency of each OMNP, $n(\%)$} \\
\hline III & $58(31)$ & $13(28)$ & 0.670 \\
\hline IV & $49(26)$ & $7(15)$ & 0.108 \\
\hline VI & $81(43)$ & $27(57)$ & 0.077 \\
\hline Other causes, $n(\%)$ & $32(17)$ & $24(51)$ & $<0.001$ \\
\hline Inflammation, $n(\%)$ & $12(6)$ & $17(36)$ & $<0.001$ \\
\hline Vascular, $n(\%)$ & $13(7)$ & $3(6)$ & 1.000 \\
\hline Neoplasm, $n(\%)$ & $6(3)$ & $3(6)$ & 0.308 \\
\hline Others, $n(\%)$ & $1(1)$ & $1(2)$ & 0.361 \\
\hline Microvascular ischemia, $n(\%)$ & $156(83)$ & $23(49)$ & $<0.001$ \\
\hline
\end{tabular}

OMNP: ocular motor nerve palsy, III: oculomotor nerve palsy, IV: trochlear nerve palsy, Vl: abducens nerve palsy.

Compared to the younger patients (age $<50$ years) with isolated OMNP $(n=47)$, patients aged $\geq 50$ years had a significantly higher frequency of microvascular ischemia ( $83 \%$ vs. $49 \%, p<0.001)$ and a lower prevalence of other causes $(17 \%$ vs. $51 \%, p<0.001$ ) (Table 5). The frequency of each OMNP did not differ between the two age groups.

\section{DISCUSSION}

This prospective study found that abducens nerve palsy was the most common (40\%) type of OMNP in neurology clinics, and that multiple OMNPs occurred in $10 \%$ of cases. The etiologies included microvascular ischemia (47\%), inflammation (21\%), stroke (5\%), trauma (5\%), neoplasm (3\%), and others (2\%), with the cause not being determined in $13 \%$ of the patients. Compared to previous retrospective studies from ophthalmology clinics, the prevalence of multiple OMNPs, vascular disorders (microvascular ischemia, stroke, CCF, and aneurysm), and inflammation was higher in our study, whereas those of trauma and undetermined cause were lower. ${ }^{9-17}$ These discrepancies in the distribution of the affected nerves and underlying etiologies may be due to differences in the study populations, designs, and inclusion criteria applied for a cause of microvascular ischemia. Vascular risk factors were defined more broadly in the present study than in previous studies. Furthermore, the entire population in this study received MRI including DWI, and we systematically assessed serum anti-GQ1b antibody levels and CSF. These procedures might have increased the prevalence of vascular and inflammatory disorders and other causes, while decreasing the proportion of cases with an undetermined cause. A lower prevalence of trauma may indicate that patients with traumatic OMNP are more likely to be managed by ophthalmologists. Our results necessitate the assessment of vascular and inflammatory causes of patients with acquired OMNP in neurological practice.

Since the possibility of secondary causes has been considered to be higher in acquired OMNP associated with neurological findings or multiple OMNPs, attention has recently been paid to isolated OMNP. Three prospective studies of Western populations found that when OMNP was isolated in patients aged $\geq 50$ years, microvascular ischemia accounted for more than $80 \%$ of cases (Table 6), ${ }^{19-21}$ with the frequency of causes other than microvascular ischemia varying from $4 \%$ to $17 \%$. Consistent with this, in our Korean population we found a frequency of causes other than microvascular ischemia of $17 \%$. However, the distribution of other causes in this study differed from that in the previous studies. In our study, the other causes were mostly vascular disorders (13/32, $41 \%)$ and inflammation $(12 / 32,38 \%)$, while common causes in the previous studies were tumors $(14 / 31,45 \%)$ and inflammation $(7 / 31,23 \%) .{ }^{19-21}$ Furthermore, the main causes of inflammation were Tolosa-Hunt syndrome and pachymeningitis in our study, and giant-cell arteritis in previous ones.

We found that the underlying causes of isolated OMNP differed significantly with patient age. The prevalence of microvascular ischemia was significantly higher in the older than the younger group ( $83 \%$ vs. $49 \%, p<0.001$ ), whereas other 
Table 6. Summary of previous and our studies on isolated OMNP

\begin{tabular}{|c|c|c|c|c|c|}
\hline & $\begin{array}{c}\text { Bendszus et al. }{ }^{18} \\
(n=43)\end{array}$ & $\begin{array}{l}\text { Chou et al. }{ }^{19} \\
\quad(n=66)\end{array}$ & $\begin{array}{c}\text { Murchison et al. }{ }^{20} \\
(n=93)\end{array}$ & $\begin{array}{c}\text { Tamhankar et al. }{ }^{21} \\
(n=109)\end{array}$ & $\begin{array}{l}\text { Our study } \\
(n=188)\end{array}$ \\
\hline Population & $\begin{array}{c}\text { Isolated 6th } \\
\text { nerve palsy (all ages) }\end{array}$ & $\begin{array}{l}\text { Isolated OMNP } \\
\text { ( } \geq 50 \text { years) }\end{array}$ & $\begin{array}{l}\text { Isolated OMNP } \\
\text { ( } \geq 50 \text { years })\end{array}$ & $\begin{array}{l}\text { Isolated OMNP } \\
\text { ( } \geq 50 \text { years })\end{array}$ & $\begin{array}{l}\text { Isolated OMNP } \\
\text { ( } \geq 50 \text { years) }\end{array}$ \\
\hline Study design & Single center & Single center & Single center & Multicenter & Multicenter \\
\hline Age, $y$, median (range) & $48(2-82)$ & $67(50-85)$ & ND & $64(50-90)$ & $60(9-88)$ \\
\hline Male, $n(\%)$ & & $40(61)$ & $66(71)$ & $66(61)$ & $122(64)$ \\
\hline \multicolumn{6}{|l|}{ Frequency of each OMNP, $n(\%)$} \\
\hline III & & $29(44)$ & $14(15)$ & $22(20)$ & $58(31)$ \\
\hline IV & & $14(21)$ & $27(29)$ & $25(23)$ & $49(26)$ \\
\hline $\mathrm{VI}$ & $43(100)$ & $23(35)$ & $52(56)$ & $62(57)$ & $81(43)$ \\
\hline Microvascular ischemia, $n(\%)$ & $10(23)$ & $57(86)$ & ND & $91(84)$ & $156(83)$ \\
\hline III & & 25 & & 19 & 43 \\
\hline IV & & 13 & & 22 & 43 \\
\hline VI & & 19 & & 50 & 70 \\
\hline Other causes, $n(\%)$ & $27(63)$ & $9(14)$ & $4(4)$ & $18(17)$ & $32(17)$ \\
\hline III & & 4 & 0 & 3 & 15 \\
\hline IV & & 1 & 1 & 3 & 6 \\
\hline VI & & 4 & 3 & 12 & 11 \\
\hline
\end{tabular}

ND: not described, OMNP: ocular motor nerve palsy, III: oculomotor nerve palsy, IV: trochlear nerve palsy, VI: abducens nerve palsy.

causes showed the opposite relationship ( $17 \%$ vs. $51 \%, p<$ 0.001 ). A recent prospective study of isolated sixth-nerve palsy found a higher incidence of other causes (63\%) and a lower incidence of microvascular ischemia (23\%), since patients of all ages were analyzed together. Our results again emphasize the importance of considering patient age when evaluating acute isolated OMNP.

The traditional guidelines based on retrospective studies of imaging patients with acute isolated OMNP have included 1) younger than 50 years, 2) pupil-involving or pupilsparing partial third-nerve palsy, 3) a history of any type of cancer, 4) any other localizing signs or symptoms, 5) no improvement within 1 month, and 6) no resolution within 3 months. ${ }^{19,20,24}$ The present study compared various clinical variables between patients with isolated OMNP due to microvascular ischemia and OMNP due to other causes. We found that only the presence and a higher intensity of headache were risk factors for causes other than microvascular ischemia, whereas the presence of vascular risk factors is more likely to be associated with microvascular ischemia. There were no intergroup differences in the proportion of cases that resolved at 1, 3, and 6 months after symptom onset, or in the frequency of a history of previous infection or cancer, pupillary involvement or pupil-sparing partial third-nerve palsy, or the degree of external ophthalmoplegia. Our results are consistent with previous studies also finding no difference in the degree of external ophthalmoplegia and pupillary involvement between cranial third-nerve palsies due to microvascular ischemia and other etiologies. ${ }^{7,19}$ Indeed, approximately one-third of patients with cranial third-nerve palsy due to microvascular ischemia are known to show some degree of pupillary involvement. ${ }^{6,24,25}$

The present study was subject to several limitations. Since the study was based on data from the neurology clinics of tertiary-care hospitals, the obtained results might not be applicable to community hospitals or ambulatory-care units. The possibility of selection bias should also be considered when interpreting the results. Microvascular ischemia is a presumptive diagnosis based on no alternative causes and spontaneous resolution with or without the presence of vascular risk factors. Since the criteria for microvascular ischemia do not entirely rule out alternative etiologies such as myasthenia gravis, a longer follow-up may be required to establish a definitive diagnosis of OMNP due to microvascular ischemia.

\section{Conflicts of Interest}

The authors have no potential conflicts of interest to disclose.

\section{Acknowledgements}

This study was supported by a grant from Korean Neurological Association (KNA-13-MI-06).

\section{REFERENCES}

1. Friedman DI. Pearls: diplopia. Semin Neurol 2010;30:54-65.

2. Danchaivijitr C, Kennard C. Diplopia and eye movement disorders. J Neurol Neurosurg Psychiatry 2004;75 Suppl 4:iv24-iv31.

3. Buracchio T, Rucker JC. Pearls and oy-sters of localization in ophthal- 
moparesis. Neurology 2007;69:E35-40.

4. Prasad S, Volpe NJ. Paralytic strabismus: third, fourth, and sixth nerve palsy. Neurol Clin 2010;28:803-833.

5. Keane JR. Fourth nerve palsy: historical review and study of 215 inpatients. Neurology 1993;43:2439-2443.

6. Jacobson DM. Pupil involvement in patients with diabetes-associated oculomotor nerve palsy. Arch Ophthalmol 1998;116:723-727.

7. Jacobson DM. Relative pupil-sparing third nerve palsy: etiology and clinical variables predictive of a mass. Neurology 2001;56:797-798.

8. Lee SH, Lee SS, Park KY, Han SH. Isolated oculomotor nerve palsy: diagnostic approach using the degree of external and internal dysfunction. Clin Neurol Neurosurg 2002;104:136-141.

9. Richards BW, Jones FR Jr, Younge BR. Causes and prognosis in 4,278 cases of paralysis of the oculomotor, trochlear, and abducens cranial nerves. Am J Ophthalmol 1992;113:489-496.

10. Rucker CW. Paralysis of the third, fourth and sixth cranial nerves. Am J Ophthalmol 1958;46:787-794.

11. Rucker CW. The causes of paralysis of the third, fourth and sixth cranial nerves. Am J Ophthalmol 1966;61:1293-1298.

12. Rush JA, Younge BR. Paralysis of cranial nerves III, IV, and VI. Cause and prognosis in 1,000 cases. Arch Ophthalmol 1981;99:76-79.

13. Tiffin PA, MacEwen CJ, Craig EA, Clayton G. Acquired palsy of the oculomotor, trochlear and abducens nerves. Eye (Lond) 1996;10:377384.

14. Berlit P. Isolated and combined pareses of cranial nerves III, IV and VI. A retrospective study of 412 patients. J Neurol Sci 1991;103:10-15.

15. Peters GB 3rd, Bakri SJ, Krohel GB. Cause and prognosis of nontraumatic sixth nerve palsies in young adults. Ophthalmology 2002;109: 1925-1928.
16. Moster ML, Savino PJ, Sergott RC, Bosley TM, Schatz NJ. Isolated sixth-nerve palsies in younger adults. Arch Ophthalmol 1984;102:13281330.

17. Park UC, Kim SJ, Hwang JM, Yu YS. Clinical features and natural history of acquired third, fourth, and sixth cranial nerve palsy. Eye (Lond) 2008:22:691-696.

18. Bendszus M, Beck A, Koltzenburg M, Vince GH, Brechtelsbauer D, Littan T, et al. MRI in isolated sixth nerve palsies. Neuroradiology 2001; 43:742-745.

19. Chou KL, Galetta SL, Liu GT, Volpe NJ, Bennett JL, Asbury AK, et al. Acute ocular motor mononeuropathies: prospective study of the roles of neuroimaging and clinical assessment. J Neurol Sci 2004;219:35-39.

20. Murchison AP, Gilbert ME, Savino PJ. Neuroimaging and acute ocular motor mononeuropathies: a prospective study. Arch Ophthalmol 2011; 129:301-305.

21. Tamhankar MA, Biousse V, Ying GS, Prasad S, Subramanian PS, Lee MS, et al. Isolated third, fourth, and sixth cranial nerve palsies from presumed microvascular versus other causes: a prospective study. Ophthalmology 2013;120:2264-2269.

22. Mollan SP, Edwards JH, Price A, Abbott J, Burdon MA. Aetiology and outcomes of adult superior oblique palsies: a modern series. Eye (Lond) 2009;23:640-644.

23. Headache Classification Committee of the International Headache Society (IHS). The International Classification of Headache Disorders, 3rd edition. Cephalalgia 2018;38:1-211.

24. Kung NH, Van Stavern GP. Isolated ocular motor nerve palsies. Semin Neurol 2015;35:539-548.

25. Keane JR. Third nerve palsy: analysis of 1400 personally-examined inpatients. Can J Neurol Sci 2010;37:662-670. 